\title{
Successful Treatment of Neoplasm-related Leg Pain with Spinal Cord Stimulator Therapy
}

\author{
Jerry P. Kalangara, $M^{1,2}$ and Vinita Singh, $M D^{1}$
}

Nearly 15.5 million people were affected by cancer in 2015 (1). Consequently, neoplasm-related pain continues to be a challenging problem that affects about $50.7 \%$ of patients in all cancer stages (2). The World Health Organization (WHO) guidelines are often used for the treatment of cancer pain, but unfortunately, 10 to $15 \%$ of these cancer patients receive suboptimal pain relief $(3,4)$. Therapy often consists of nonsteroidal antiinflammatory drugs, opioids, and adjuvant medications (such as gabapentinoids, tricyclic anti-depressants), but medication management can be limited by undesired side effects (3). For these refractory cases, there needs to be alternative treatment modalities. Here, we present a case of a patient with cancer related pain of his leg successfully treated with spinal cord stimulator (SCS) therapy. Since the patient was deceased at the time of writing this case report, we were unable to obtain informed consent. We thus sought approval from our local IRB, who determined that approval is not required.

\section{CASE PRESENTATION}

A 68 year old male with a history of Parkinson's disease and stage IV melanoma that has relapsed into his left psoas muscle presented with continuous left leg pain for 8 months. He had a history of multiple pelvic lymph node dissections and radiation treatment to his left leg (Fig. 1). The pain was rated as $7-9 / 10$ on the pain numeric rating scale (NRS) and described as deep, sharp, and aching. Physical exam was remarkable for left leg lymphedema

From : 'Department of Anesthesiology, Emory University School of Medicine, Atlanta, $\mathrm{GA} ;{ }^{2}$ Atlanta Veterans Affairs Medical Center, Atlanta, GA

Author for correspondence: Vinita Singh, MD

Address: Department of Anesthesiology, Emory University School of Medicine, 550 Peachtree Rd. NE, Atlanta, GA 30308

E-mail: Vinita.Singh@emory.edu (from previous pelvic lymphadenectomy) as well as erythema and induration in left groin near the femoral crease. The painful area was not tender to palpation and exhibited no signs of allodynia, hyperalgesia, skin temperature changes, hair changes, or atrophy. He did not have any signs of lumbar radiculopathy on exam. His motor exam demonstrated weakness in left hip flexion and knee extension, graded as 4/5. There was painful numbness along the medial aspect of the left thigh (nondermatomal). Bilateral patellar and achilles tendon reflex were within normal limits.

He trialed multiple medications for pain control including: hydrocodone, oxycodone, gabapentin, duloxetine, which were either ineffective or intolerable due to side effects. His pain was poorly controlled with his medication regimen of acetaminophen $650 \mathrm{mg}$ as needed, fentanyl transdermal patch $25 \mathrm{mcg}$ per hour, hydromorphone $1 \mathrm{mg} 6$ to 8 times per day, and topical lidocaine $5 \%$ patches. Based on a history and physical exam, the patient's pain appeared to have both nociceptive and neuropathic components and likely due to the neoplasm itself and possible surgery and radiation-induced nerve damage. Intrathecal opioid drug delivery system was considered, but since the patient had mild allergic reactions (rash) to multiple opioids, an alternate treatment plan was explored in an attempt to address neuropathic features of his pain. Initially, a lumbar sympathetic block was performed at L3 level, which provided $50 \%$ pain relief; however, this only lasted for 24 hours. The patient was not interested in undergoing a series of injections and a SCS trial was proposed to address his predominant neuropathic pain, which responded to the lumbar sympathetic block.

Magnetic resonance imaging (MRI) compatible SCS was chosen given his history of cancer and possible need for future MRI. He underwent SCS trial with dual lead (Medtronic Inc.) placement at the top of T10 vertebrae for 5 days. Two leads were used to ensure 


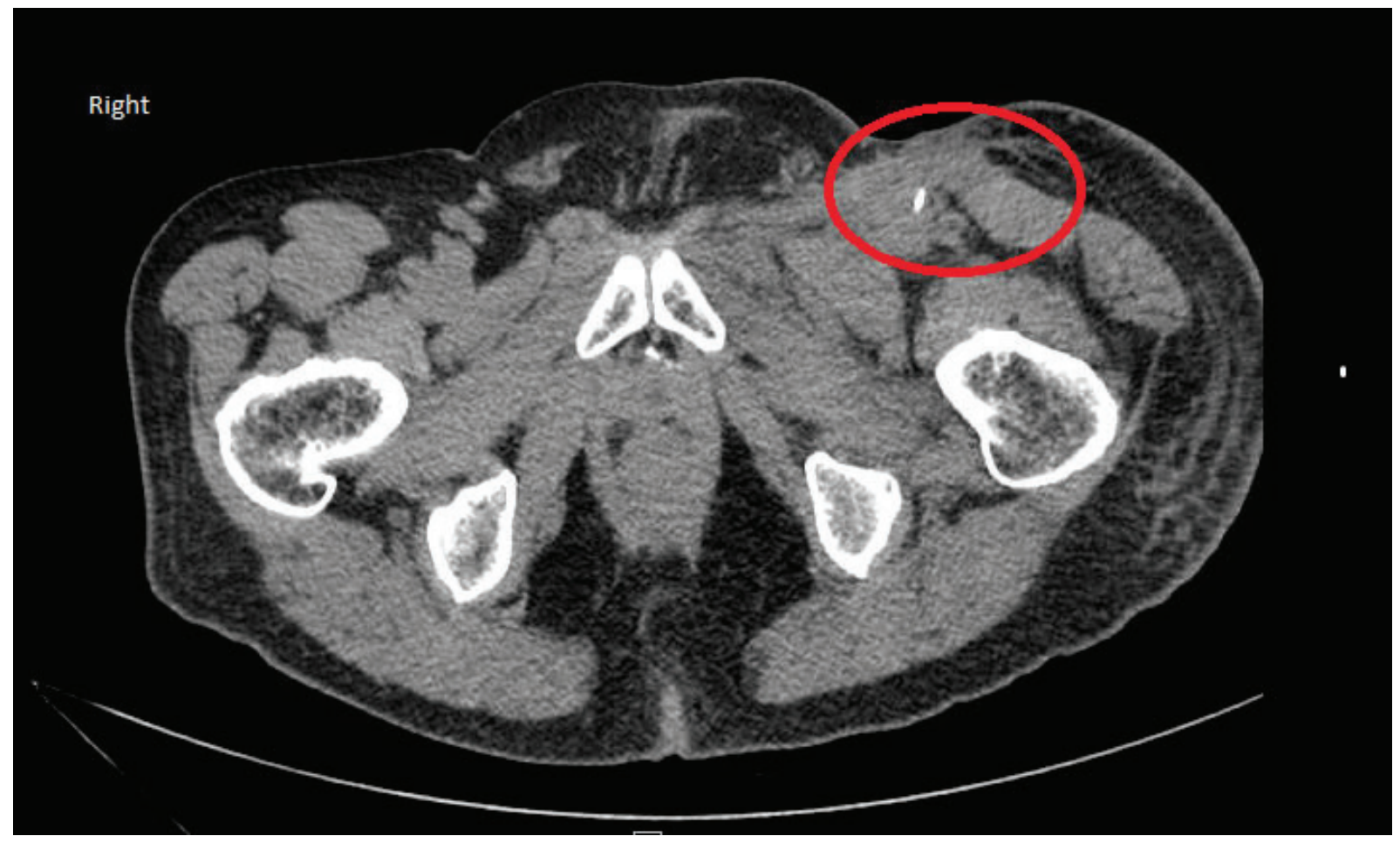

Fig. 1. CT scan of abdomen and pelvis, axial image through inguinal region showing scarring of the surrounding soft tissue in left groin.

adequate coverage and to provide more flexibility in the future if the pain pattern broadened with disease progression. This provided adequate paresthesia coverage over his painful areas, including left groin and anterior thigh. He reported $75 \%$ pain relief and improved activity with SCS trial. His pain score with activity (worst pain score) was $3 / 10$ and without activity (best pain score) was $1 / 10$. He was able to attend church, which he had not previously due to an inability to walk in from the parking lot.

He then underwent permanent implantation of SCS with dual octrode leads placed at the previous location (Fig. 2). Following implantation, he reported $85 \%$ pain relief and reduced his fentanyl patch dose by $50 \%$. $\mathrm{He}$ also reported improved function and participated in more activities. His pain remained well controlled with fentanyl patch $12.5 \mathrm{mcg}$ per hour and SCS at 5 months. Unfortunately, his disease burden worsened (with left thigh subcutaneous metastasis, multiple lung metastasis, and brain metastasis) and he developed failure to thrive. Additionally, he developed anemia requiring repeated transfusions secondary to his underlying illness of metastatic melanoma and possible medication effect (immunotherapy). His pain continued to be located in left groin and anterior thigh and was fairly controlled at 8 months with his SCS, but required additional fentanyl patch $(37.5 \mathrm{mcg}$ per hour). Further, he had fatigue and weakness (from disease progression and anemia) as well as increasing tremors (from Parkinson's disease) leading to unsteady gait/frequent falls, which limited his quality of life. He entered a hospice program for end of life care at 8.5 months and expired 11 months after the SCS placement.

\section{DISCUSSION}

Cancer pain is often seen as a mixed pain type with both neuropathic and somatic-nociceptive components $(5,6)$. This neuropathic pain component can represent 15 to $40 \%$ of chronic cancer pain (7). Further, neuropathic pain often responds poorly to opioid therapy and there is a real need for developing alternate treatment strategies. 
SCS therapy is a growing technology that has been shown to successfully treat multiple pain conditions, including complex regional pain syndrome, lumbar radicular pain, post-laminectomy syndrome, and refractory angina/ peripheral vascular disease (8). SCS is an established therapeutic modality that has the potential for application in cancer pain. Thus far, SCS has only been scantly used for this population and represents an avenue that needs to be further explored. There have been a handful of reports describing successful pain relief in intractable chemotherapy-induced peripheral neuropathy $(9,10)$. In our case report, we demonstrate the effective use of SCS for pain relief of neoplasm-related leg pain. Not only did the patient demonstrate a long lasting reduction in NRS score by over $50 \%$, but he also reported increased functionality and improved quality of life, which are critical with the management of terminal disease. Part of the reluctance to undertake SCS for cancer pain may stem from cost concerns in patients with a terminal disease. The estimated cost of SCS placement is approximately $\$ 25,000$. However, neoplasm-related pain has been treated with intrathecal drug delivery system using opioids and other medications for several years and this strategy can entail expenses of over $\$ 10,000$ for the pump itself. When taking into consideration added hospital stay (for inpatient intrathecal/epidural catheter trial and observation after pump implant, given a risk of respiratory depression with initiation of intrathecal opioids), subsequent medication refills and follow-up visits, the incremental costs are even greater. Despite this, multiple

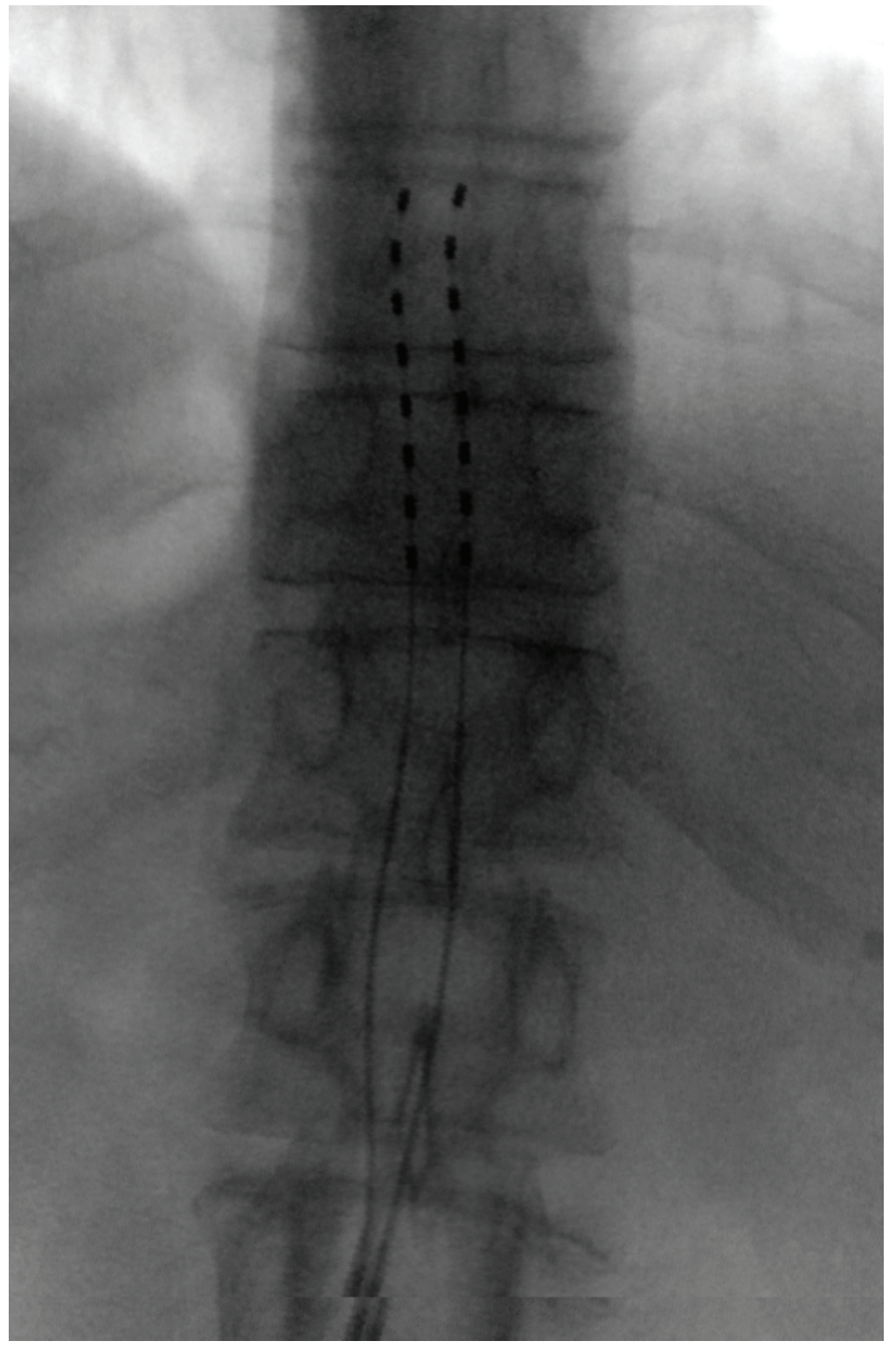

Fig. 2. Final SCS leads.

studies have demonstrated a cost effective profile of this modality in cancer pain patients with a life expectancy of at least 6 months $(11,12)$. This patient survived for 11 months after implant. Further, SCS therapy has fewer significant adverse effects associated with it when compared to intrathecal pumps.

In addition, the average baseline health care cost among opioid treated patients is $\$ 17,120$ with follow-up costs of $\$ 42,829$ in one estimate (13). Direct cost estimates are unavailable for pain related health care in cancer patients. However, inadequate pain control in 
this population is a major contributor to emergency department visits, frequent hospitalizations, and utilization of skilled nursing facilities (14). In particular, our patient avoided pain related hospitalizations for up to 6 months, likely justifying the cost of the SCS therapy. Thus, SCS therapy should at least be considered if it may have some potential benefit in these patients with recalcitrant neoplasm-related pain especially with neuropathic pain component. Due to the myriad of variables in the treatment of cancer pain, further research is needed to substantiate and validate the long-term feasibility and safety of SCS for cancer-related pain.

\section{REFERENCES}

1. American Cancer Society. Cancer treatment and survivorship, facts and figures 2016-2017; https://www.cancer.org/content/ dam/cancer-org/research/cancer-facts-and-statistics/cancertreatment-and-survivorship-facts-and-figures/cancer-treatmentand-survivorship-facts-and-figures-2016-2017.pdf. Accessed 09/27, 2017.

2. van den Beuken-van Everdingen, $\mathrm{M} H \mathrm{HJ}$, Hochstenbach, L MJ, Joosten, E AJ, Tjan-Heijnen, V CG, Janssen, D JA. Update on Prevalence of pain in patients with cancer: Systematic review and meta-analysis. Journal of Pain and Symptom Management 2016; 51:1070-1090.

3. Flagg A 2nd, McGreevy K, Williams K. Spinal cord stimulation in the treatment of cancer-related pain: "Back to the origins". Curr Pain Headache Rep 2012; 16:343-349.

4. Sloan P, Melzack R. Long-term patterns of morphine dosage and pain intensity among cancer patients. Hospice ] 1999; 14:3547.

5. Peng L MS, Zejun Z, Wei K, Bennett MI. Spinal cord stimulation for cancer-related pain in adults. Cochrane Database of Systematic Reviews 2015.

6. Ro LS, Chang $\mathrm{KH}$. Neuropathic pain: Mechanisms and treatments. Chang Gung Med J 2005; 28:597-605.

7. Berger A, Dukes E, Mercadante S, Oster G. Use of antiepileptics and tricyclic antidepressants in cancer patients with neuro- pathic pain. Eur ] Cancer Care (Engl) 2006; 15:138-145.

8. Lee AW, Pilitsis JG. Spinal cord stimulation: Indications and outcomes. Neurosurg focus 2006; 21:E3.

9. Abd-Elsayed A SN, Sachdeva H. Efficacy of spinal cord stimulators in treating peripheral neuropathy: A case series. Journal of Clinical Anesthesia 2016; 28:74-77.

10. Cata JP, Cordella JV, Burton AW, Hassenbusch SJ, Weng HR, Dougherty PM. Spinal cord stimulation relieves chemotherapy-induced pain: A clinical case report. J Pain Symptom Manage 2004; 27:72-78.

11. Miguel R. Interventional treatment of cancer pain: The fourth step in the World Health Organization analgesic ladder? Cancer Control 2000; 7:149-156.

12. Knight $\mathrm{KH}$, Brand FM, McHaourab AS, Veneziano G. Implantable intrathecal pumps for chronic pain: Highlights and updates. Croat Med ] 2007; 48:22-34.

13. Xie L, Joshi AV, Schaaf D, Mardekian J, Harnett J, Shah ND, Baser O. Differences in health care utilization and associated costs between patients prescribed vs. nonprescribed opioids during an inpatient or emergency department visit. Pain Pract 2014; 14:445-456.

14. Elsayem AF, Elzubeir HE, Brock PA, Todd KH. Integrating palliative care in oncologic emergency departments: Challenges and opportunities. World J Clin Oncol 2016; 7:227-233. 